\title{
ESTUDO FITOQUÍMICO DAS CASCAS DO CAULE DE DUGUETIA GLABRIUSCULA - ANNONACEAE, BIOMONITORADO PELO ENSAIO DE TOXICIDADE FRENTE A ARTEMIA SALINA LEACH
}

João Máximo de Siqueira*, Maria Graziela Ziminiani

DFB, CCBS, Universidade Federal de Mato Grosso do Sul, CP 549, 79070-900 Campo Grande - MS

Ubirazilda Maria Resende

DBI, CCBS, Universidade Federal de Mato Grosso do Sul, CP 549, 79070-900 Campo Grande - MS

Maria Amélia Diamantino Boaventura

Departamento de Química, ICEx, Universidade Federal de Minas Gerais, CP 702, 31270-901 Belo Horizonte - MG

Recebido em 3/3/00; aceito em 2/8/00

\begin{abstract}
ACTIVITY-GUIDED ISOLATION OF THE CONSTITUENTS FROM BARK OF STEM OF DUGUETIA GLABRIUSCULA - ANNONACEAE, USING BRINE SHRIMP LETHALITY TEST (BSL). The extract obtained from stem bark of Duguetia glabriuscula - Annonaceae was evaluated by Brine Shrimp Lethality test (BSL). The bioactive compounds, oxobufoline and lanuginosine, two oxoaporphine alkaloids were isolated by activity-guided fractionation. In addition, the compounds asaraldehyde, (+)-allo-aromadendrane-10ß,14-diol, and two aporphine alkaloids, polyalthine and oliveridine were also obtained.
\end{abstract}

Keywords: Annonaceae; Duguetia glabriuscula; Artemia salina; oxoaporphine alkaloids.

\section{INTRODUÇÃO}

Dando continuidade ao estudo fitoquímico biomonitorado das espécies nativas da família Annonaceae em Mato Grosso do Sul com a utilização do ensaio de toxicidade frente a Artemia salina $^{1}$, TAS, serão apresentados os dados referentes à espécie Duguetia glabriuscula. O TAS, por ser um ensaio biológico rápido, de baixo custo e simples tem sido amplamente utilizado e demonstrando uma boa correlação com a atividade antitumoral ${ }^{2}$, sendo então indicado na avaliação preliminar de extratos vegetais ${ }^{3}$.

Na família Annonaceae, o gênero Duguetia está representado, até o momento, por 70 espécies, mas não está entre os mais estudados fitoquimicamente. O estudo fitoquímico tem se concentrado nos gêneros Annona (120 espécies catalogadas), Goniothalamus (115), Monodora (20), Rollinia (65), Uvaria (150) e Xylopia $(100-150)^{4}$. Diversos alcalóides já foram isolados de várias espécies de Duguetia ${ }^{5}$ e, pelo estudo fitoquímico biomonitorado de D. panamensis, utilizando o ensaio de toxicidade frente a Artemia salina, foi obtida como substância ativa o 2,4,5-trimetoxilestireno (1), porém apresentando baixa toxicidade frente a esse microcrustáceo e também fraca atividade antitumoral ${ }^{6}$

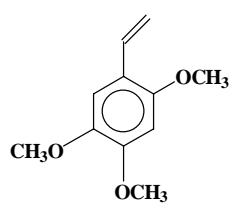

(1)

Duguetia glabriuscula é um arbusto de pouco exemplares em Mato Grosso do Sul. Desta espécie vegetal foi isolado, inicialmente, do extrato hexânico das folhas e agora também isolado das cascas do caule, o (+)-10ß,14-allo-aromadendranodiol, (2), essa substância apresentou-se inativa para o microcrustáceo ${ }^{7}$,

\footnotetext{
*E-mail: jmaximo@nin.ufms.br
}

mas com atividade antimicrobiana considerável ${ }^{8}$. Devido a abundância do referido sesquiterpeno, o mesmo foi submetido a algumas modificações na porção funcional da molécula, conduzindo a produtos de baixa atividade antimicrobiana e com atividade frente a Artemia salina, ainda que pouco significativa ${ }^{7}$.

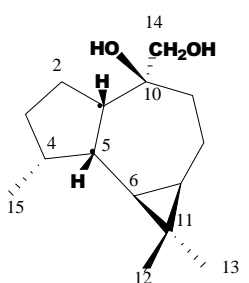

(2)

O objetivo do presente trabalho é o isolamento de substâncias biologicamente ativas do extrato etanólico $95 \%$ ativo, em água, obtido das cascas do caule de Duguetia glabriuscula Annonaceae, pelo estudo cromatográfico biomonitorado, utilizando a Artemia salina como organismo teste.

\section{RESULTADOS E DISCUSSÃO}

O extrato etanólico $95 \%$ das cascas do caule de D. glabriuscula apresentou atividade sobre o microcrustáceo, TAS, $\mathrm{DL}_{50}=131,7(76,3-227,5)^{\dagger} \mu \mathrm{g} / \mathrm{mL}$. Este extrato então foi submetido a partição entre $\mathrm{CHCl}_{3}$ e $\mathrm{MeOH} / \mathrm{H}_{2} \mathrm{O}$. A atividade se concentrou na fração clorofórmica (TAS, $\mathrm{DL}_{50}=56,6 \mu \mathrm{g} / \mathrm{mL}$ ), como descrito na Tabela 1 .

Essa fração ativa foi fracionada em coluna, da qual foram obtidas os asaraldeído (3), oxobuxifolina (4), (+)-10,14 $\beta$-alloaromadendranodiol (2), descrito anteriormente ${ }^{8}$, lanuginosina (5), polialtina (6) e oliveridina (7).

A substância 3 apresentou aspecto de um sólido branco amorfo. $\mathrm{O}$ espectro de $\mathrm{RMN}$ de ${ }^{1} \mathrm{H}$ exibe dois sinais simples 
Tabela 1. Teste de toxicidade para a Artemia salina das frações e substâncias isoladas das cascas do caule de D. glabruscula.

\begin{tabular}{ccc}
\hline & $\mathrm{DL}_{50}$ & $\begin{array}{c}\text { intervalo de } \\
\text { confiança de 95\% } \\
(\mu \mathrm{g} / \mathrm{mL})\end{array}$ \\
\hline Cascas do caule & $(\mu \mathrm{g} / \mathrm{mL})$ & \\
Extrato etanólico & 131,7 & $76,5-227,5$ \\
Fração clorofórmica & 56,6 & $45,4-70,7$ \\
Asaraldeído (3) & $>1000$ & - \\
Oxobuxifolina (4) & 10,4 & $8,2-13,3$ \\
Lanuginosina (5) & 27,6 & $15,0-50,6$ \\
Polialtina (6) & $>1000$ & - \\
Oliveridina (7) & $>1000$ & - \\
\hline
\end{tabular}

relativos a hidrogênios aromáticos em $\delta 7,31$ e $\delta$ 6,48, três sinais de metoxilas aromáticas em $\delta 3,96,3,91$ e 3,86 e um sinal em $\delta 10,30$, relacionado ao hidrogênio formila.

$\mathrm{O}$ espectro RMN de ${ }^{13} \mathrm{C}$ de $\mathbf{3}$, juntamente com os sinais encontrados no espectro de DEPT 135, evidenciam três sinais intensos relativos as metoxilas aromáticas em $\delta 56,19 ; \delta 56,21$ e $\delta 56,28$. Seis sinais relativos a carbonos aromáticos, aparecem em $\delta 95,90,108,98,117,31,143,55,155,64,158,77$ e o sinal em $\delta 188,10$ do carbono formila ${ }^{9}$. Foi obtido o mapa de contorno HMQC que confirma as correlações observadas para os hidrogênios aromáticos e formila dessa substância. O EM-IE é caracterizado por um pico de íon molecular intenso (sendo também o pico base) a m/z 196 e por um pico $\mathrm{M}^{+}-1$ (34\%).

As substâncias 4 e 5, derivados de alcalóides oxaporfínicos, possuem espectros no RMN ${ }^{1} \mathrm{H}$ e ${ }^{13} \mathrm{C}$ muito semelhantes. Uma outra observação importante é que quando adicionada à essas substâncias uma solução de ácido clorídrico a 5\%, observa-se a formação de cor avermelhada ${ }^{5}$.

Os espectros de RMN de ${ }^{1} \mathrm{H}$ de 4 e 5 exibem, em cada um deles, 2 sinais de hidrogênios aromáticos $(\delta 8,15$ e 8,85, em 4 e $\delta$ 7,77 e 8,75 em 5) com constantes de acoplamentos, J, igual 5,2 $\mathrm{Hz}$, comum em hidrogênios $\alpha$ e $\beta$ do anel piridínico (anel B), em $\mathrm{C}-4$ e C-5 ${ }^{10}$, respectivamente. A diferença do deslocamento químico, $\delta$ para os hidrogênios em C-4 $(\delta 8,15$ para 4 e 7,77 para 5) é devido a presença de uma metoxila em C-3 (anel A) de $\mathbf{4}$, que provoca um efeito $\gamma$-gauche sobre $\mathrm{H}-4$ deslocando-o para maiores valores de $\delta$. A mesma substituição não é observada em $\mathbf{5}$ e é confirmada pela presença de um singleto em $\delta 7,09$, relativo ao H-3, completando o padrão de substituição de 4 e 5 nesse anel. Observa-se também um sinal simples referente aos hidrogênios do grupo metilenodioxi em $\delta 6,44$ em 4 e 6,35 em 5. Mais três sinais de hidrogênios aromáticos, juntamente com um outro sinal de metoxila aparecem em ambas as substâncias e foram atribuídos ao anel D e pelo deslocamento da metoxila e multiplicidade dos hidrogênios, foi possível determinar o padrão de substituição em 4 e 5 (vide dados experimentais).

Semelhanças espectrais também são observadas para as substâncias 6 e 7, uma vez que são derivados de alcalóides aporfínicos7-oxigenados. Basicamente, observa-se o mesmo padrão para as duas substâncias: a presença do grupo metilenodioxi, em C-1/C2 , que devido à torção do anel no sistema bifenílico, aparece como dupleto $(\delta 6,05$ e 5,92 em $\mathbf{6}$ e 6,08 e 5,96 em $7, \mathrm{~J}=1,2$
$\mathrm{Hz})^{5}$; a presença de um singleto $(\delta 6,54)$ em 7 relativo ao hidrogênio em C-3 e para 6, nessa mesma posição, observa-se um singleto de metoxila $(\delta 4,01)$. Uma informação importante que pode ser obtida no espectro de $\mathrm{RMN}{ }^{1} \mathrm{H}$ para esse tipo de alcalóide 7-oxigenado é a definição da estereoquímica cis ou trans dos hidrogênios H-6a e H-7. Na espécie Duguetia spixiana, dentre outras espécies da família Annonaceae ${ }^{5}$, os alcalóides 7-oxigenados apresentam configuração trans e o sinal do espectro é constituído de dupletos com constantes de acoplamento de 9 a $12 \mathrm{~Hz}$ (mais comum, $12 \mathrm{~Hz})^{11,12}$. Na estereoquímica cis, o $J$ observado foi de 2 a $3 \mathrm{~Hz}$, como descrito para alcalóides isolados do gênero Stephania (Menispermaceae) ${ }^{5}$. O padrão de substituição no anel $\mathrm{D}$, caracterizado em ambas moléculas pela presença de um substituinte em C-9 (metoxila) e os deslocamentos químicos e multiplicidade observados para de $\mathrm{H}-8, \mathrm{H}-10$ e $\mathrm{H}-11$.

A substância 2 foi descrita anteriormente ${ }^{8}$.

De todas as substâncias citadas a atividade sobre a Artemia salina convergiu para as $\mathbf{4}$ e $\mathbf{5}$, como demonstrado na Tabela 1 .

O estudo fitoquímico biomonitorado das cascas do caule de $D$. glabriuscula convergiu para as frações ativas ao microcrustáceo das quais foram isolados representantes dos alcalóides oxaporfínicos. Os oxaporfínicos têm sido avaliados em diferentes atividades biológicas, entre elas: atividade citotóxica ${ }^{13-15}$, anti-agregante plaquetário ${ }^{16}$, antimicrobiana e antifúngica ${ }^{17}$, e tem sido sugerido, para esse grupo de alcalóides, a predominância da atividade citotóxica ${ }^{16}$. Assim, se o TAS tem como interesse convergir para substâncias com atividade citotóxica, presente em um determinado extrato, o estudo fitoquímico biomonitorado das cascas do caule de D. glabriuscula foi efetivo, pois foram isoladas substâncias como a oxobuxifolina e a lanuginosina, ativas ao TAS e merecedoras de testes mais específicos ${ }^{3}$.

\section{PARTE EXPERIMENTAL}

Procedimentos Experimentais Gerais: Os espectros de RMN de ${ }^{1} \mathrm{H}$ e ${ }^{13} \mathrm{C}$, de 300 e $75 \mathrm{MHz}$, respectivamente, foram registrados em espectrômetro DPX-300-Bruker. Os deslocamentos químicos, $\delta$, relativos ao sinal do tetrametilsilano $(\delta=0,0)$. Os espectros de massas foram obtidos no espectrômetro de massas TRIO-1000 através de injeção direta. Os solventes e reagentes utilizados tiveram grau de pureza P.A. Para as cromatografias em camada delgada (CCD) analítica e preparativa utilizou-se sílica-gel 60 G MERCK.

Ensaio biológico: O ensaio de toxicidade sobre a Artemia salina (TAS) foi executado de acordo com a metodologia proposta por Meyer ${ }^{2}$. Diluições das amostras e do controle positivo foram feitos pelo método de diluições aritméticas em solução aquosa de sal marinho sintético (38g/L, Enterprise Co.) com 1\% DMSO (v/ v). Todas as etapas foram acompanhadas utilizando-se o sulfato de quinidina como controle positivo ${ }^{2}$ e cada avaliação foi repetida pelo menos duas vezes. Utilizou-se o método Probitos de análise para obtenção das $\mathrm{DL}_{50}$ e respectivos intervalos de confiança. Os extratos foram considerados ativos quando TAS <1000ppm.

Material vegetal: As cascas do caule de Duguetia glabriuscula R. E. Fries (R. E. Fries) - Annonaceae - foram coletadas no município de Jardim/MS. A amostra botânica foi identificada pelo Prof R. de Mello-SiIva. A exsicata foi depositada no Herbário da UFMS (Campo Grande, Mato Grosso do Sul) sob o número 4769.

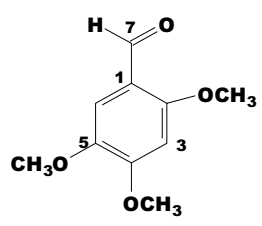

(3)

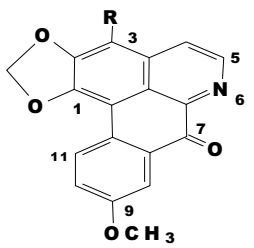

(4) $\mathrm{R}=\mathrm{OCH}_{3}$ (5) $\mathbf{R}=\mathbf{H}$

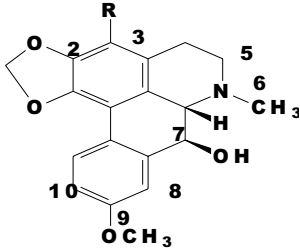

(6) $\mathrm{R}=\mathrm{OCH}_{3}$

(7) $\mathbf{R}=\mathbf{H}$ 
Obtenção dos extratos vegetais: As cascas do caule foram extraídas exaustivamente com etanol $95 \%$ por maceração a $40^{\circ} \mathrm{C}$, concentrados à pressão reduzida e testados (vide Tabela 1). Esse extrato foi então solubilizado em metanol:água 9:1 e submetido a partição entre clorofórmio. A fração clorofórmica, ativa, foi fracionada em coluna cromatográfica (sílica gel, $\mathrm{CHCl}_{3}: \mathrm{CH}_{3} \mathrm{OH}$-gradiente).

Asaraldeído ou 2,4,5-Trimetoxi-benzaldeído (3): Sólido branco amorfo. I.V. V $v_{\text {máx }}\left(\mathrm{KBr}, \mathrm{cm}^{-1}\right): 2962,2920,2850,1660,1609$, $1520,1479,1292,1219,1129,1027,864,823$. RMN ${ }^{1} \mathrm{H}:(300$ $\mathrm{MHz}, \delta): 10,30(\mathrm{H}-7) ; 7,31$ (H-6) e 6,48 (H-3) (s, $1 \mathrm{H}$ cada); 3,$96 ; 3,91 ; 3,86$ (s, 3H cada). $\mathrm{RMN}{ }^{13} \mathrm{C}(75 \mathrm{MHz}, \delta) 188,10(\mathrm{C}-$ 7); 158,64 (C-2); 155,77 (C-4); 143,55 (C-5); 117,31 (C-1); 108,98 (C-6); 95,90 (C-3); 56,28; 56,21; 56,19 (metoxilas). EMIE m/z (int. rel.): $197[\mathrm{M}+1]^{++}(10) ; 196(100) ; 195[\mathrm{M}-1]^{+}$ (34); 181 (68); 150 (53); 125 (84); 69 (91).

Oxobuxifolina (4): Sólido microcristalino alaranjado. U.V $\gamma\left(\mathrm{CH}_{3} \mathrm{OH}, \mathrm{nm}\right) 245,281,320$ (ombro), 382, 457; [ $+\mathrm{HCl}, 1 \mathrm{~N}, 1$ gota]; 265, 283, 320 ombro, 408, 530. RMN de ${ }^{1} \mathrm{H}$ (DMSO-d $\mathrm{D}_{6}$, $300 \mathrm{MHz}$ ): 8,85 (d, $J=5,2 \mathrm{~Hz}, \mathrm{H}-5), 8,48$ (d, $J=8,8 \mathrm{~Hz}, \mathrm{H}-$ 11), 8,15 (d, $J=5,2 \mathrm{~Hz}, \mathrm{H}-4), 7,77$ (d, $J=3,0 \mathrm{~Hz}, \mathrm{H}-8), 7,43$ (dd, $J=8,8$ e $3,0 \mathrm{~Hz}, \mathrm{H}-10), 6,44$ (s, $\mathrm{OCH}_{2} \mathrm{O}$ em C-1/C-2), 4,21 e 3,93 $\left(\mathrm{OCH}_{3}\right.$ em $\mathrm{C}-3$ e $\mathrm{C}-9$, respectivamente). $\mathrm{RMN}$ de ${ }^{13} \mathrm{C}$ (DMSO-d 6 , $\left.75 \mathrm{MHz}\right): 180,90(\mathrm{C}-7), 158,61$ (C-9), 149, 28 (C-1), 146,10 (C-6), 144,11 (C-5), 137,54 (C-2), 134,93 (C3), 131,63 (C-11a), 130,20 (C-3a), 128,24 (C-11), 126,04 (C7a), 124,70 (C-11b), 122,18 (C-10), 121,36 (C-11c), 119,01 $(\mathrm{C}-4), 109,75(\mathrm{C}-8), 103,29\left(\mathrm{OCH}_{2} \mathrm{O}\right.$ em $\left.\mathrm{C}-1 / \mathrm{C}-2\right), 60,27$ $\left(\mathrm{OCH}_{3}\right), 55,48\left(\mathrm{OCH}_{3}\right.$ em C-9). EMIE, m/z (int. rel.) $\mathrm{M}^{+} 335$ $(100), 320\left[\mathrm{M}^{+}-\mathrm{CH}_{3}\right](73 \%), 305\left[\mathrm{M}^{++}-\mathrm{CH}_{2} \mathrm{O}\right](36 \%)$.

Lanuginosina (5): Sólido microcristalino alaranjado.RMN 1H: $\left(300 \mathrm{MHz}, \mathrm{CDCl}_{3} / \mathrm{MeOD}\right) \delta: 8,75(\mathrm{~d}, J=5,2 \mathrm{~Hz}, \mathrm{H}-5)$; $8,43(\mathrm{~d}, J=8,9 \mathrm{~Hz}, \mathrm{H}-11) ; 7,90(\mathrm{~d}, J=2,9 \mathrm{~Hz}, \mathrm{H}-8) ; 7,77$ $(\mathrm{d}, J=5,2 \mathrm{~Hz}, \mathrm{H}-4) ; 7,23$ (dd, $J=8,9$ e $J=2,9 \mathrm{~Hz}, \mathrm{H}-10)$; 7,09 (s, H-3); 6,35 (s, $\mathrm{OCH}_{2} \mathrm{O}$ em C-1/C-2) e 3,96 (s, $\mathrm{OCH}_{3}$ em C-9). EMIE, m/z (int. rel.) $\mathrm{M}^{++} 305$ (100), $304\left[\mathrm{M}^{++}-1\right]$ (82), $290\left[\mathrm{M}^{+}-\mathrm{CH}_{3}\right](11)$.

Polialtina (6): sólido amorfo castanho: U.V $\lambda\left(\mathrm{CH}_{3} \mathrm{OH}, \mathrm{nm}\right)$ $215 ; 239 ; 283 ;[+\mathrm{NaOH}, 01 \mathrm{~N}, 1$ gota] sem alteração. RMN de ${ }^{1} \mathrm{H}\left(\mathrm{CDCl}_{3}, 300 \mathrm{MHz}\right) 7,88(\mathrm{~d}, J=8,6 \mathrm{~Hz}, \mathrm{H}-11), 7,29$ (sl, H8), 6,84 (dd, $J=8,6$ e 2,4 Hz, H-10), 6,05 e 5,92 (dupletos, $J$ $=1,4 \mathrm{~Hz}, \mathrm{OCH}_{2} \mathrm{O}$ em C-1/C-2), 4,63 (d, $\left.J=12,2 \mathrm{~Hz}, \mathrm{H}-6 \mathrm{a}\right)$, 4,01 e 3,85 (s, $\mathrm{OCH}_{3}$ em C-3 e C-9, respectivamente), 3,65 (d, $J=12,2 \mathrm{~Hz}, \mathrm{H}-7), 2,65\left(\mathrm{~s}, \mathrm{NCH}_{3}\right) . \mathrm{EMIE}, \mathrm{m} / \mathrm{z}$ (int. rel.) 355 (90), $337\left[\mathrm{M}^{+} \cdot-\mathrm{H}_{2} \mathrm{O}\right](27), 324\left[\mathrm{M}^{+} \cdot \mathrm{OCH}_{3}\right]$ (38) e $312\left[\mathrm{M}^{+}\right.$$\left.\mathrm{CH}_{2} \mathrm{NCH}_{3}\right](31), 43(100 \%)$.

Oliveridina (7): sólido amorfo castanho: U.V $\lambda\left(\mathrm{CH}_{3} \mathrm{OH}\right.$, nm) $215 ; 239 ; 283 ;$ [+NaOH, $01 \mathrm{~N}, 1$ gota] sem alteração. RMN de ${ }^{1} \mathrm{H}\left(\mathrm{CDCl}_{3}, 300 \mathrm{MHz}\right):$ 7,94 (d, $\left.J=9,0 \mathrm{~Hz}, \mathrm{H}-11\right), 7,34$ (dl, $J=2,0 \mathrm{~Hz}, \mathrm{H}-8), 6,87$ (dd, $J=9,0$ e 2,0 Hz, H-10), 6,54 (s, H3), 6,08 e 5,96 (dupletos, $J=1,2 \mathrm{~Hz}, \mathrm{OCH}_{2} \mathrm{O}$ em C-1/C-2), $4,73$ (d, $J=12,5 \mathrm{~Hz}, \mathrm{H}-6 \mathrm{a}), 3,65$ (d, $J=12,5 \mathrm{~Hz}, \mathrm{H}-7), 3,86$ (s, $\mathrm{OCH}_{3}$ em C-9), 2,79 (s, $\left.\mathrm{NCH}_{3}\right) . \mathrm{RMN}$ de ${ }^{13} \mathrm{C}\left(\mathrm{CDCl}_{3}, 75 \mathrm{MHz}\right)$ : 159,16 (C-9), 147,6 (C-2), 141,14 (C-7a), 140,60 (C-1), 127,54 (C-11), 127,23 (C-3a), 121,98 (C-3b), 121,38 (C-11a), 116,48 (C-11b), 113,40 (C-10), 109,11 (C-8), 106,66 (C-3), 100,80 $\left(\mathrm{OCH}_{2} \mathrm{O}\right.$ em C-1/C-2), 69,62 (C-7), 64,39 (C-6a), 59,57 $\left(\mathrm{OCH}_{3}\right.$ em C-9), 48,90 (C-5), 40,00 $\left(\mathrm{NCH}_{3}\right), 22,75$ (C-4).

\section{AGRADECIMENTOS}

À PROPP/UFMS pelo apoio financeiro. Aos Depto de Química/CCET/UFMS (docentes, corpo técnico) e Chemistry Departament of University of Liverpool - UK por terem gentilmente facilitado as análises das substâncias aqui descritas (RNM ${ }^{1} \mathrm{H}^{13} \mathrm{C}$, IV e Espectros de massas, respectivamente). À Maria da Conceição Guerra de Souza (Lab. de Farmacognosia, DFB, CCBS, UFMS) pelo apoio técnico durante a execução da parte experimental desse trabalho.

\section{REFERÊNCIAS}

1. Siqueira, J. M. de; Pereira, N. F.; Bomm, M. D.; Garcez, W. S.; Boaventura, M. A. D.; Quim. Nova 1998, 21, 557.

2. Meyer, B. N.; Ferrigni, N. R.; Putnam, J. E.; Jacobsen, L. B.; Nichols, D. E. J; McLaughlin, J. L.; Planta Medica 1982, 45, 31 .

3. McLaughlin, J. L.; Chang, C-J.; Smith, D. L.; Simple Bench-Top Bioassays (BS \& PD) for Discovery of Plant Antitumor Compounds - Review of Recent Progress In Human Medicinal Agents from Plants, Ed. Kinghorn \& Balandrini, Nova Iorque, 1993, p. 112-137.

4. Leboeuf, M.; Cave, A.; Bhaumik, P. K.; Mukherjee, B.; Mukherjee, R.; Phytochemistry 1982, 21, 2783.

5. Cavé, A.; Leboeuf, M.; Waterman, P. G. In Alkaloids: Chemical and Biological Perspectives, Ed. S. William Pelleiter (John Wiley \& Sons), v. 5, cap. 3, New York, 1987, p.133-627.

6. Wang, Z-W.; Ma, W-W.; McLaughlin, J. L.; Gupta, M. P.; J. Nat. Prod. 1988, 51, 382.

7. De Lima, D. P.; Beatriz, A.; Ramos, A. A.; De Siqueira, J. M.; De Oliveira, C. C.; Marques, R.; Quim. Nova 1997, 20, 616.

8. Siqueira, J. M.; Boaventura, M. A. D.; Garcez, F.; Garcez, W. S.; Oliveira, C. C.; Fitoterapia 1997, 68, 89.

9. Pouchert, C. J.; Behnke, J. In The Aldrich Library of $13 C$ and $1 H$ FT-NMR, Ed. Aldrich Chemical Cia, Inc.,1993, 2,969 .

10. Silverstein, R. M.; Bassler, G. C.; Morrill, T. C. Identificação Espectrométrica de Compostos Orgânicos, 5a. Ed, Guanabara Koogan ed., Rio de Janeiro, 1994.

11. Debourges, D.; Roblot, F.; Hocquemiller, R.; Cavé, A.; J. Nat. Prod. 1987, 50, 664.

12. Razamizafy, S.; Hocquemiller, R.; Cavé, A.; Fournet, A.; J. Nat. Prod. 1987, 50, 674.

13. Sonnet, P. E.; Jacobson, M.; J. Pharm. Sci. 1971, 60, 1254.

14. Wu, Y-C.; Chang, G-Y.; Yih, C.; Duh, C-Y.; Wang, SK.; Phytochemistry 1993, 33, 497.

15. Wijeratne, E. M. K.; Gunatilaka, A. A. L.; Kingston, D. G. I.; Haltiwanger, R. C.; Eggleston, D. S.; Tetrahedron 1995, 51, 7877 .

16. Chen, K-S.; Ko, F-N.; Teng, C-W.; Wu, Y-C.; Planta Medica 1996, 62, 133.

17. Hufford, C. D.; Sharma, A. S.; Oguntinein, B. O.; J. Pharm. Sci. 1980, 69, 1180.

18. Finney, D. J.; Probit Analysis, A Statistical Treatment of the Sigmoid Response Curve. University Press, Cambridge, 1974.

19. Ferrigni, N. R.; McLaughlin, J. L.; Powell, R. G.; Smith, JR., C. R.; J. Nat.Prod. 1984, 47, 347. 